TITLE:

\title{
Effects of confinement on the statistics of encounter times: exact analytical results for random walks in a partitioned lattice
}

$\operatorname{AUTHOR}(\mathrm{S}):$

Kalay, Ziya

\section{CITATION:}

Kalay, Ziya. Effects of confinement on the statistics of encounter times: exact analytical results for random walks in a partitioned lattice. Journal of Physics A: Mathematical and Theoretical 2012, 45(21): 215001.

ISSUE DATE:

2012-06-01

URL:

http://hdl.handle.net/2433/156176

\section{RIGHT:}

C IOP Publishing 2012:; この論文は出版社版でありません。引用の際 には出版社版をご確認ご利用ください。; This is not the published version. Please cite only the published version. 


\title{
Effects of confinement on the statistics of encounter times: exact analytical results for random walks in a partitioned lattice
}

\author{
Ziya Kalay \\ Institute for Integrated Cell-Material Sciences (WPI-iCeMS), Kyoto University, \\ Kyoto, 606-8501, Japan \\ E-mail: zkalay@icems.kyoto-u.ac.jp
}

\begin{abstract}
We study the effects of temporarily and permanently confining domains on the statistics of first-passage times in finite lattices in one and two dimensions. We present exact results for the mean and variance of the first-passage time between arbitrary sites in: (1) a finite one-dimensional lattice partitioned into temporarily confining domains, and (2) a finite two-dimensional lattice with reflecting boundaries for a single random walker and an immobile target. In the one-dimensional case, we also present the full first-passage time distribution via numerical inversion of Laplace transforms.
\end{abstract}

PACS numbers: 02.50.-r, 05.40.-a, 66.30.-h

Submitted to: J. Phys. A 


\section{Introduction}

Distribution of the time at which an event occurs for the first time is of great relevance for stochastic processes. Calculation of this distribution, commonly known as the firstpassage time distribution [1], is almost a standard procedure while investigating the kinetics of reactions between randomly moving particles [2, 3], exciton dynamics in crystals including traps [4], the exit time of a particle out of a region with a given potential energy landscape [5], estimating the time to failure [6], determining the optimal strategy for searching targets [7], and more [1]. Here we would like to emphasize the role of first-passage time analysis in chemical kinetics in structured environments.

Seminal works by Smoluchowski [2] and Noyes [3] have inspired much of the work done in the study of bimolecular reactions. Smoluchowski's formulation, given in an early theoretical investigation on the kinetics of colloid coagulation [2], involves integrating the diffusion equation with the initial and boundary conditions chosen such that there is an infinite amount of available reactants which react immediately at an interaction distance. These kind of reactions are called diffusion controlled reactions as the rate of the reaction only depends on the rate at which the particles find each other. In Noyes' formulation [3], a counterpart of the system of interest is considered, in which the reactants are not consumed upon encounter. The central quantity in this theory is the probability of first reencounter between non-reactive particles, from which reaction rates in the original system can be calculated. Both of these theories have been extended to more general cases in a large number of subsequent works $[8,9,10,11,12]$.

In addition to studies in continuum, there is a significant body of work on bimolecular reactions between random walkers in a lattice $[13,14,15,16,17,18,19,20]$. In the simplest case, the system consists of only two random walkers one of which is stationary. For this case, and for periodic boundary conditions, the average time it takes for one of the particles to find the other and its variance was first calculated by Montroll and Weiss in their seminal first passage time analysis for lattice walks [21].

Recently, effects of structured space, especially molecular confinement, on reaction kinetics began to emerge as an important concept. For instance, Condamin et al. [22] presented general results for first-passage times in complex media as a function of confinement volume and the initial configuration of particles, Bénichou et al. [23] emphasized the importance of geometrical constraints on kinetics of reactions between randomly moving (anomalous or simple diffusion) molecules. In a related work, Meyer et al. [24] discussed the identification of universalities in the characteristics of firstpassage time distributions in confining domains, in detail. Condamin et al. [25, 26] also presented a novel and elegant approach for calculating the statistics of first-passage times in bounded geometries by starting from the Kac's formula [27], which relates the number of steps required to return to the starting point in an irreducible graph (see ref. [26] for a rederivation of this result).

General results for the first-passage time distributions when the searcher and the target are in the same confining domain were obtained earlier [28, 24]. For 
instance, very recently, Tejedor et al. [29] obtained practical analytical results on the first encounter time distribution for two random walkers in a finite one-dimensional space with absorbing or reflecting boundaries. However, an analytical result in the presence of multiple, connected, temporarily confining domains where the searcher can get temporarily trapped in a confining domain has not been found. Note that this physical picture is extremely relevant for reactions occurring in complex systems such as a biological cell, where the medium is partitioned into many compartments by permeable boundaries such that the molecules are temporarily trapped.

In this study we would like to contribute to this subject by providing exact solutions to the following two problems: 1) First-passage time to a given site, starting from an arbitrary site, in a finite 1D (one-dimensional) lattice partitioned into temporarily confining domains, which is relevant for interacting random walkers when one of the walkers is fixed; 2) first-passage time to a given site in a 2D (two-dimensional) finite lattice with reflecting boundaries. These problems are frequently encountered while studying the time between dissociation and association events in reversible reactions.

By providing these results, our main goal is to contribute to the efforts of developing a thorough understanding of reaction kinetics in confined spaces, which is crucial for the study of complex systems such as a cell. Moreover, we would like to emphasize the importance of considering higher order moments of the encounter time distribution, if not the full distribution, in characterising the temporal pattern of reactions, rather than focusing on the average reaction rates.

In the next section, we give a brief explanation of how the encounter rate of particles, or the first-passage time to encounter following an encounter at $t=0$, is related to the time between reactions. In Section 3, we provide the solutions of the first-passage time problems stated above, with their derivations. Lastly, we give a discussion of our results, our conclusions, and a future outlook in Section 4.

\section{Reaction rate and encounter statistics}

The rate at which particles encounter each other strongly depends on the properties of the medium where they reside. For freely diffusing particles, the time between encounters is controlled by the diffusion coefficient and particle concentration alone. However, for particles that are temporarily trapped in a finite region, the encounter time also depends on the characteristic time each particle spends in a confining region.

In most cases, chemical reactions do not occur during the first diffusive encounter between the reactants and require many subsequent encounters. Luckily, it is possible to formulate the problem for such activation controlled reactions by considering the distribution of times between two subsequent encounters alone.

Suppose that a pair of particles react with probability $\omega$ upon an encounter. If these molecules have just separated after a non-reactive encounter, they will react with 
probability $h(t) d t$ at time $t$, where $h(t)$ is given by [3].

$$
\begin{aligned}
h(t) & =\omega f_{\mathrm{enc}}(t)+\omega(1-\omega) \int_{0}^{t} d t^{\prime} f_{\mathrm{enc}}\left(t-t^{\prime}\right) f_{\mathrm{enc}}\left(t^{\prime}\right) \\
& +\omega(1-\omega)^{2} \int_{0}^{t} d t^{\prime \prime} f_{\mathrm{enc}}\left(t-t^{\prime \prime}\right) \int_{0}^{t^{\prime \prime}} d t^{\prime} f_{\mathrm{enc}}\left(t^{\prime \prime}-t^{\prime}\right) f_{\mathrm{enc}}\left(t^{\prime}\right) \\
& +\ldots,
\end{aligned}
$$

where $f_{\text {enc }}(t)$ is the distribution of the time it takes until the next encounter (involving either of the dissociated particles) given the particles dissociated at $t=0$, i.e. the distribution of first reencounter times. Note that the terms on the right hand side of equation (1) correspond to the probability that the pair of particles react at time $t$ after the first, second, third and larger number of encounters. Moments of the reaction time distribution can be expressed in terms of the Laplace transform of $f_{\text {enc }}(t)$

$$
\left\langle t_{\mathrm{rxn}}^{n}\right\rangle=(-1)^{n} \lim _{\epsilon \rightarrow 0} \frac{d^{n} \widetilde{h}(\epsilon)}{d \epsilon^{n}}=(-1)^{n} \lim _{\epsilon \rightarrow 0} \frac{d^{n}}{d \epsilon^{n}} \sum_{m=0}^{\infty} \omega(1-\omega)^{m}\left[\widetilde{f}_{\mathrm{enc}}(\epsilon)\right]^{m+1},
$$

where $\epsilon$ is the Laplace transform variable, tildes distinguish Laplace transformed quantities, and we used the relation between the derivatives of the Laplace transform of a function and its moments [30]. Specifically, the average time between reactions, and its variance are given by

$$
\begin{aligned}
& \mu_{\mathrm{rxn}}=\left\langle t_{\mathrm{rxn}}\right\rangle=\frac{\mu_{\mathrm{enc}}}{\omega}, \\
& \sigma_{\mathrm{rxn}}^{2}=\left\langle t_{\mathrm{rxn}}^{2}\right\rangle-\left\langle t_{\mathrm{rxn}}\right\rangle^{2}=\frac{\omega \sigma_{\mathrm{enc}}^{2}+(1-\omega) \mu_{\mathrm{enc}}^{2}}{\omega^{2}},
\end{aligned}
$$

where $\mu_{\text {enc }}$ and $\sigma_{\text {enc }}^{2}$ are the mean and variance of $f_{\text {enc }}(t)$, respectively.

\section{Statistics of first-passage times in specific lattices}

In this section, we first recall the well-known mathematical relation between the firstpassage time distribution and the probability propagator for a random walk, and subsequently present our results for specific cases in one and two dimensions.

Let $P\left(t, \vec{r} ; 0, \vec{r}_{0}\right)$ be the probability of finding a random walker at the site $\vec{r}$ at time $t$ provided that it was initially at the site $\vec{r}_{0}$. This probability can be expressed as the product of the probability of visiting $\vec{r}$ at time $s<t$ for the first time, and staying there for a time $t-s$, such that [1]

$$
P\left(t, \vec{r} ; 0, \vec{r}_{0}\right)=\int_{0}^{t} d s Q\left(s, \vec{r} ; 0, \vec{r}_{0}\right) P(t-s, \vec{r} ; 0, \vec{r}),
$$

where $Q\left(s, \vec{r} ; 0, \vec{r}_{0}\right)$ is the probability that a random walker visits the site $\vec{r}$ for the first time, provided that it started at $\vec{r}_{0}$. Taking the Laplace transform of both sides in equation (2) and rearranging terms, we get

$$
\widetilde{Q}\left(\epsilon, \vec{r} ; t=0, \vec{r}_{0}\right)=\int_{0}^{\infty} d t e^{-\epsilon t} Q\left(t, \vec{r} ; 0, \vec{r}_{0}\right)=\frac{\widetilde{P}\left(\epsilon, \vec{r} ; t=0, \vec{r}_{0}\right)}{\widetilde{P}(\epsilon, \vec{r} ; t=0, \vec{r})} .
$$


Note that the $n^{\text {th }}$ moment of the first-passage time distribution $Q(t)$ can be conveniently calculated through the following relation

$$
\left\langle t_{\overrightarrow{r_{0}, \vec{r}}}^{n}\right\rangle=\int_{0}^{\infty} d t t^{n} Q(t)=(-1)^{n} \lim _{\epsilon \rightarrow 0} \frac{d^{n}}{d \epsilon^{n}} \widetilde{Q}(\epsilon) .
$$

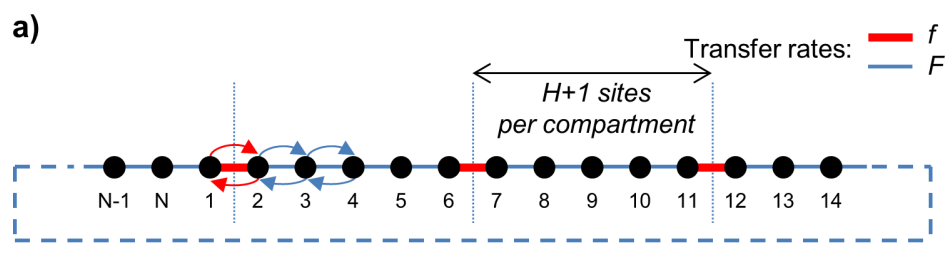

b) 1D finite periodic lattice transformed into a bounded chain

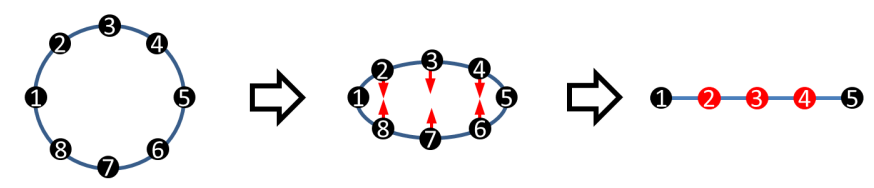

c) $2 \mathrm{D}$ torus transformed into a rectangle

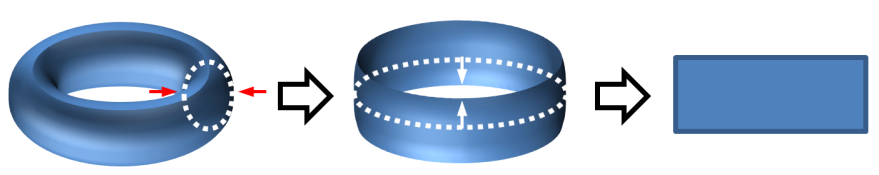

Figure 1. a. Schematic illustration of the $1 \mathrm{D}$ partitioned lattice. A periodic lattice, or a ring, of $N$ sites that is partitioned into domains of $H+1$ sites. Thicker (red) bonds between lattice sites have a lower transfer rate than those of the thinner (blue), and act as barriers that hinder diffusion. The dashed (blue) line is drawn to emphasize the periodicity of the lattice. b. Illustration of how a $1 \mathrm{D}$ finite periodic lattice is transformed into a chain of sites with reflective boundaries via equation (40). The arrows (red) point between sites whose occupancy are summed up. Note that the boundary points are not affected from the transformation. c. Illustration of how a torus, representing a $2 \mathrm{D}$ periodic lattice, is transformed into a rectangular region with reflective boundaries through equation (41). Note that this procedure consists of applying the transformation in a twice, and the occupancies of a maximum of 4 sites may be summed up.

\subsection{One-dimensional lattice partitioned into domains}

Perhaps the simplest idealized problem by which the effects of temporarily confining domains, or compartments, can be studied is random walk in a $1 \mathrm{D}$ periodic lattice that is partitioned into domains by bonds with lower transition rates, as illustrated in figure 1(a). The probability of finding a particle in such a lattice obeys a Master equation [31]

$$
\begin{aligned}
\frac{d P_{m}(t)}{d t} & =P_{m+1}(t)+P_{m-1}(t)-2 P_{m}(t) \\
& -\Delta \sum_{r}\left(P_{r+1}(t)-P_{r}(t)\right)\left(\delta_{m, r}-\delta_{m, r+1}\right),
\end{aligned}
$$


where the walker only jumps between neighbouring lattice sites, $\Delta=1-f / F, F$ and $f$ are the transition rates between sites in the same and adjacent compartments, respectively, $t$ is dimensionless time $(t=F$. time), and $r$ denotes the index of a site to the left of a compartment boundary, such that the summation is over a subset of all lattice sites. Laplace transform of the probability, denoted by $\widetilde{P}_{m}(\epsilon)$, and its derivative can be obtained analytically with the help of the results given in reference [31], and are found to be

$$
\begin{aligned}
& \widetilde{P}_{m}(\epsilon)=\widetilde{\eta}_{m}(\epsilon)-\Delta \sum_{r} X_{r}(\epsilon) f_{m-r}(\epsilon) \\
& \frac{d \widetilde{P}_{m}(\epsilon)}{d \epsilon}=\frac{d \widetilde{\eta}_{m}(\epsilon)}{d \epsilon}-\Delta \sum_{r}\left[\left(Z_{r}(\epsilon)+Y_{r}(\epsilon)\right) f_{m-r}(\epsilon)+X_{r}(\epsilon) \frac{d f_{m-r}(\epsilon)}{d \epsilon}\right],
\end{aligned}
$$

where

$$
A X=g, \quad A Y=\frac{d g}{d \epsilon}, \quad A^{2} Z=-\frac{d A}{d \epsilon} g
$$

and

$$
\begin{aligned}
\widetilde{\psi}_{m}(\epsilon) & =\frac{1}{N} \sum_{k=1}^{N} \frac{e^{-i(2 \pi k / N) m}}{\epsilon+2(1-\cos (2 \pi k / N))} \\
\widetilde{\eta}_{m}(\epsilon) & =\widetilde{\psi}_{m-m_{0}}(\epsilon) \\
f_{m}(\epsilon) & =\widetilde{\psi}_{m}(\epsilon)-\widetilde{\psi}_{m-1}(\epsilon) \\
g_{m}(\epsilon) & =\widetilde{\eta}_{m+1}(\epsilon)-\widetilde{\eta}_{m}(\epsilon) \\
A_{m, n} & =(1-\Delta) \delta_{m, n}+\epsilon \Delta \psi_{r_{n}-r_{m}} \\
\frac{d A_{m, n}}{d \epsilon} & =\Delta\left(\psi_{r_{n}-r_{m}}+\epsilon \frac{d \psi_{r_{n}-r_{m}}}{d \epsilon}\right)
\end{aligned}
$$

where $A$ is a square matrix with $N /(H+1)$ rows, $r_{k}=1+(H+1)(k-1)$ is the position of the site to the left of the $k^{\text {th }}$ barrier and $k$ runs from 1 to $N /(H+1)$, each compartment contains $H+1$ lattice sites where $H$ is an even number greater that 0 , and $\psi_{m-m_{0}}(t)$ is the Green's function, or the probability propagator, for a periodic translationally invariant lattice of $N$ sites [32]. The function $\eta_{m}(t)$ is the probability of finding the random walker at the $m^{\text {th }}$ site in the absence of any barriers, i.e. $\Delta=0$. We consider a localized initial condition, such that the particle starts at the site $m_{0}$, which is reflected by equation (10). Overall, the problem of obtaining $\widetilde{P}_{m}(\epsilon)$ is reduced to solving a set of linear systems of equations defined in equation (8), where each system contains $N /(H+1)$ equations.

In the limit $\epsilon \rightarrow 0$, the linear systems in equation (8) can be solved exactly such that the probability and its derivative are given by

$$
\begin{aligned}
\lim _{\epsilon \rightarrow 0} \widetilde{P}_{m}(\epsilon) & =\lim _{\epsilon \rightarrow 0}(N \epsilon)^{-1}+\widetilde{\eta}_{m}^{\mathrm{f}}(0)-G_{1}\left(g, f ; m_{0}, m\right), \\
\lim _{\epsilon \rightarrow 0} \frac{d \widetilde{P}_{m}(\epsilon)}{d \epsilon} & =-\lim _{\epsilon \rightarrow 0}\left(N \epsilon^{2}\right)^{-1}+\widetilde{\eta}_{m}^{f \prime}(0) \\
& -G_{1}\left(g^{\prime}, f ; m_{0}, m\right)-G_{1}\left(g, f^{\prime} ; m_{0}, m\right)-G_{2}\left(h, f ; m_{0}, m\right),
\end{aligned}
$$


with

$$
\begin{aligned}
G_{1}\left(x, y ; m_{0}, m\right) & =\frac{\Delta}{\beta} \sum_{r} x_{r}\left(0 ; m_{0}\right) y_{m-r}(0) \\
& +\frac{\Delta^{2}}{(1-\Delta) \beta} \sum_{r}\left(\frac{x_{r}\left(0 ; m_{0}\right)}{H+1}-\frac{x^{*}\left(0 ; m_{0}\right)}{N}\right) y_{m-r}(0), \\
G_{2}\left(x, y ; m_{0}, m\right) & =\frac{\Delta}{\beta^{2}} \sum_{r} x_{r}\left(0 ; m_{0}\right) y_{m-r}(0)+\left\{\left(\frac{2 \Delta^{2}}{(1-\Delta) \beta^{2}}+\frac{\Delta^{3}}{(1-\Delta)^{2} \beta^{2}(H+1)}\right)\right. \\
& \left.\times \sum_{r}\left(\frac{x_{r}\left(0 ; m_{0}\right)}{H+1}-\frac{x^{*}\left(0 ; m_{0}\right)}{N}\right) y_{m-r}(0)\right\}
\end{aligned}
$$

and $\eta_{m}^{\mathrm{f}}={\widetilde{\psi_{m-m_{0}}^{\mathrm{f}}}}_{\mathrm{\psi}_{m}^{\mathrm{f}}}$ being the finite part of $\psi_{m}(\epsilon)$ as $\epsilon$ goes to 0 , i.e. without the $k=N$ term in the definition given in equation (9), and

$\beta=1-\Delta+\Delta /(H+1), \quad h_{s}=\sum_{r} \widetilde{\psi}_{r-s} g_{r}, \quad g^{*}=\sum_{r} g_{r}, \quad h^{*}=\sum_{r} h_{r}$

where $s$ denotes a site to the left of a barrier and it takes on all the values that $r$ can take. Note that the Laplace transform of the probability and its derivative diverge as $\epsilon \rightarrow 0$. However, the mean and variance of first reencounter times remain finite, and are given by

$\mu_{m_{0}, m}=N\left(\widetilde{P}_{m, m}^{\mathrm{f}}(0)-\widetilde{P}_{m_{0}, m}^{\mathrm{f}}(0)\right)$,

$\sigma_{m_{0}, m}^{2}=2 N\left(N \widetilde{P}_{m, m}^{\mathrm{f}}(0)\left(\widetilde{P}_{m, m}^{\mathrm{f}}(0)-\widetilde{P}_{m_{0}, m}^{\mathrm{f}}(0)\right)+\left.\frac{d \widetilde{P}_{m_{0}, m}^{\mathrm{f}}}{d \epsilon}\right|_{0}-\left.\frac{d \widetilde{P}_{m, m}^{\mathrm{f}}}{d \epsilon}\right|_{0}\right)-\mu_{m_{0}, m}^{2}$,

where the superscript $f$ denotes the finite part of the corresponding quantities as $\epsilon \rightarrow 0$, and $m_{0}$ in $\widetilde{P}_{m_{0}, m}^{\mathrm{f}}(0)$ denotes the initial position. The exact forms of $\mu_{m_{0}, m}$ and $\sigma_{m_{0}, m}^{2}$ can be calculated by using equations (11) and (12), which yields

$$
\begin{aligned}
\mu_{m_{0}, m} & =N\left[\widetilde{\eta}_{m}^{\mathrm{f}}(0 ; m)-\widetilde{\eta}_{m}^{\mathrm{f}}\left(0 ; m_{0}\right)\right]-N\left[G_{1}(g, f ; m, m)-G_{1}\left(g, f ; m_{0}, m\right)\right], \\
\sigma_{m_{0}, m}^{2} & =N^{2}\left[\mu_{0}\left(m_{0}, m\right)\left(\widetilde{\eta}_{m}^{\mathrm{f}}(0 ; m)+\widetilde{\eta}_{m}^{\mathrm{f}}\left(0 ; m_{0}\right)-G_{1}(g, f ; m, m)-G_{1}\left(g, f ; m_{0}, m\right)\right)\right] \\
& +2 N\left[\widetilde{\eta}_{m}^{\mathrm{f}}(0 ; m)-\widetilde{\eta}_{m}^{\mathrm{f} \prime}\left(0 ; m_{0}\right)-\left(G_{1}\left(g^{\prime}, f ; m, m\right)+G_{1}\left(g, f^{\prime} ; m, m\right)\right.\right. \\
& \left.\left.+G_{2}(h, f ; m, m)-G_{1}\left(g^{\prime}, f ; m_{0}, m\right)-G_{1}\left(g, f^{\prime} ; m_{0}, m\right)-G_{2}\left(h, f ; m_{0}, m\right)\right)\right],
\end{aligned}
$$

where the primes denote differentiation with respect to $\epsilon$. These results are valid for any initial condition specified by $m_{0}$ and $m$. As the main quantity of our interest is the reencounter time, the initial separation between the particles will always be equal to 1 , such that the mean and variance of the reencounter time are given by

$$
\begin{aligned}
& \mu_{\mathrm{r}}=\frac{1}{2 H} \sum_{\left|m-m_{0}\right|=1}^{\prime} \mu_{m_{0}, m}, \\
& \sigma_{\mathrm{r}}^{2}=\frac{1}{2 H} \sum_{\left|m-m_{0}\right|=1}^{\prime} \sigma_{m_{0}, m}^{2},
\end{aligned}
$$


where the primed summation indicates that $m$ and $m_{0}$ are in the same compartment. The expressions look complicated; however, some of the summations in $G_{i}(m, m)-$ $G_{i}\left(m_{0}, m\right)$ drop out depending on the values of $m$ and $m_{0}$. Whenever the particles start in the same compartment, we have

$$
\begin{aligned}
& G_{1}(g, f ; m, m)-G_{1}\left(g, f ; m_{0}, m\right)=\frac{\Delta}{\beta} \sum_{r}\left[g_{r}(0 ; m)-g_{r}\left(0 ; m_{0}\right)\right] f_{m-r}(0), \\
& G_{1}\left(g, f^{\prime} ; m, m\right)-G_{1}\left(g, f^{\prime} ; m_{0}, m\right)=\frac{\Delta}{\beta} \sum_{r}\left[g_{r}(0 ; m)-g_{r}\left(0 ; m_{0}\right)\right] f_{m-r}^{\prime}(0), \\
& G_{2}(h, f ; m, m)-G_{2}\left(h, f ; m_{0}, m\right)=\frac{\Delta}{\beta} \sum_{r}\left[h_{r}(0 ; m)-h_{r}\left(0 ; m_{0}\right)\right] f_{m-r}(0) .
\end{aligned}
$$

Note that all of these terms stay finite as $\Delta \rightarrow 1$. If $m_{0}$ and $m$ happen to lie in different compartments, equations (21) and (22) contain a multiple of $(1-\Delta)^{-1}$ and equation (23) retains an even more strongly diverging term proportional to $(1-\Delta)^{-2}$. However the term proportional to $(1-\Delta)^{-1}$ in $G_{1}\left(g^{\prime}, f ; m, m\right)-G_{1}\left(g^{\prime}, f ; m_{0}, m\right)$ does not vanish whether $m_{0}$ and $m$ are in the same compartment or not. Therefore, the expressions containing this term diverge as $\Delta \rightarrow 1$. As a result, if the particles start in different compartments, the mean encounter time diverges as $(1-\Delta)^{-1}$ with increasing confinement strength, whereas its variance diverges as $(1-\Delta)^{-2}$. If the particles start in the same compartment, the mean encounter time is always finite regardless of confinement strength; however, the variance still diverges with $(1-\Delta)^{-1}$.

In order to display the effects of confinement strength, it is convenient to define the normalized mean and variance of the return time as

$$
\begin{aligned}
& \mu_{\mathrm{rn}}=\mu_{\mathrm{r}} / \mu_{\mathrm{r}, \mathrm{per}}, \\
& \sigma_{\mathrm{rn}}^{2}=\sigma_{\mathrm{r}}^{2} / \sigma_{\mathrm{r}, \mathrm{per}}^{2},
\end{aligned}
$$

where $\mu_{\mathrm{r} \text {,per }}$ and $\sigma_{\mathrm{r} \text {,per }}^{2}$ are the mean and variance of the return time in a periodic lattice that does not include any barriers (see equations (49) and (50) of the next section regarding $\mu_{\text {r,per }}$ and $\sigma_{\text {r,per }}^{2}$ ). In figure 2 , we display the behaviour of the normalized mean and variance as a function of confinement strength, $\Delta$, for a lattice with $N=25$ and $H=4$.

It is interesting to note that when the random walker and the target start in the same compartment, and as the transfer rate between two compartments goes to zero, the variance of the return time increases without bound even if the lattice is finite. As the confinement strength increases, the random walker rarely escapes from the first compartment, which includes the target, without encountering the target first. Even though these escape without encounter events are rare, their impact on the return time distribution is significant due to the lengthy excursion the random walker may have to take after the escape, and may lead the second moment of the return time distribution to diverge.

In order to gain insight into how the variance of the return time can diverge in a finite lattice in a more quantitative manner, let us consider a simplified problem. 


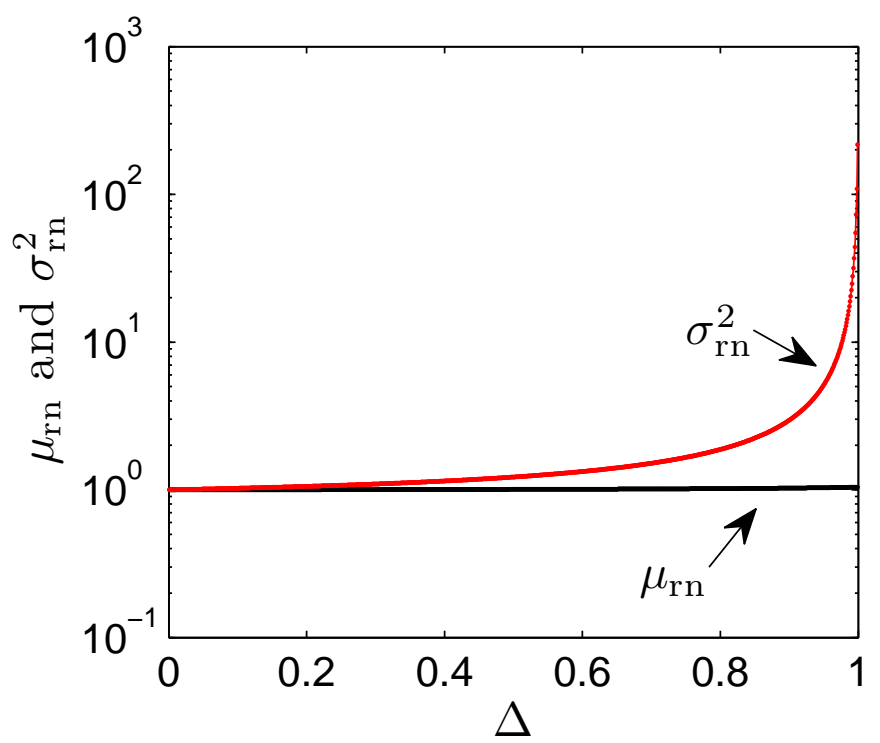

Figure 2. Normalized mean and variance of the return time as a function of confinement strength $\Delta$, given by equations (24) and (25). Note that the normalized mean return time is practically unaffected by the confinement strength; whereas its variance diverges as $\Delta \rightarrow 1$. For this plot, $N=25$ and $H=4$.

Consider a periodic lattice with $N$ compartments, and suppose that the transfer rate between each compartment is equal to $f$, and that the confinement strength is high, such that $f \simeq 0$. Since the random walker cannot transfer between compartments easily, it will be uniformly distributed over an empty compartment before it escapes, and the distribution of the escape time can be well approximated by an exponential distribution $f e^{-f t}$ (also see ref. [33]). Therefore, we can think of each compartment as a lattice site where the random walker hops between adjacent sites at a rate $f$. We are interested in calculating the mean and variance of the return time, that is the time it takes for the random walker to find the target if they are placed in the same compartment at $t=0$. Starting from this configuration, suppose that the random walker moves to another compartment without ever finding the target with probability $P_{\text {fug }}$, and it finds the target without ever leaving the compartment with probability $1-P_{\text {fug }}$. Therefore, the distribution of return times can be separated into two parts

$$
P_{\mathrm{r}}(t)=\left(1-P_{\text {fug }}\right) P_{0}(t)+P_{\text {fug }} P_{1}(t ; f),
$$

where $P_{0}(t)$ is the return time distribution given the random walker stays in the initial compartment until it finds the target, and $P_{1}(t ; f)$ is the distribution of the return time if the random walker left the initial compartment without encountering the target first. We do not assume a specific form for $P_{0}(t)$, but we use the fact that it does not depend on $f$. If the confinement strength is high, i.e. $f \simeq 0, P_{\text {fug }}$ can be approximated by $f / f_{\mathrm{r}}$, where $f_{\mathrm{r}}^{-1}$ is the average time it takes for the random walker to find the target, given it can never escape the compartment. The calculation of $P_{\text {fug }}$ involves defining a random variable that is positive (negative) when the escape takes place before an encounter, and 


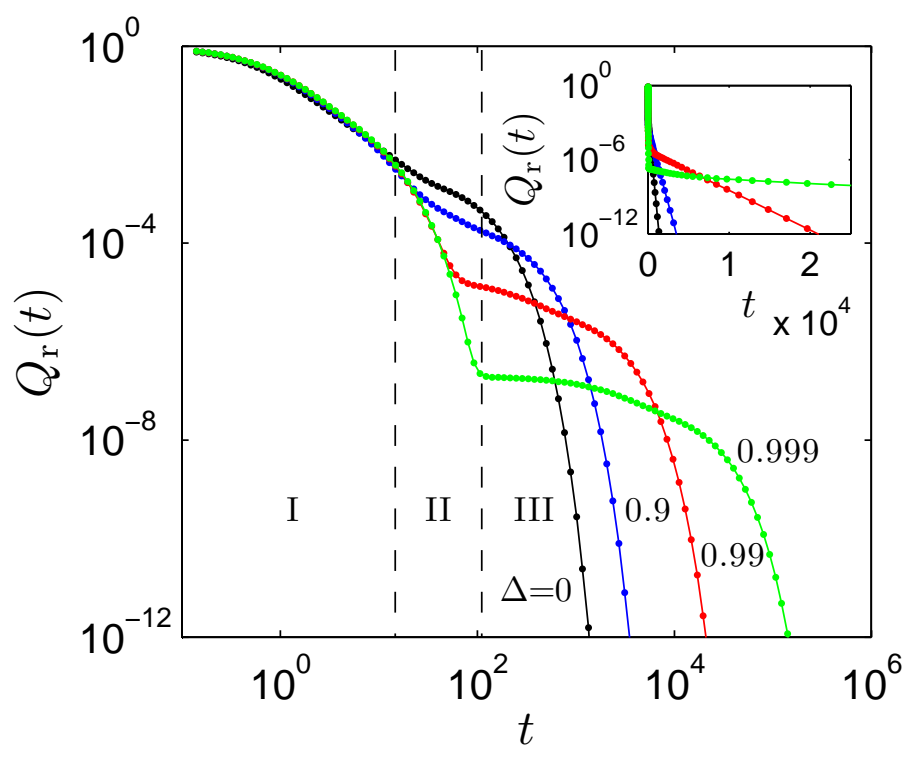

Figure 3. Distribution of the return time for different confinement strengths $\Delta$, obtained by numerical inversion of the Laplace transform $\widetilde{Q}(\epsilon)$ (see equation (33)) and averaging over adjacent random walker-target configurations. In all cases, we considered the parameter values $N=25$, and $H=4$. Plots in the main figure are shown on $\log -\log$ axes, and display the features of the return time distribution for different confinement strengths. The numbers placed near the curves indicate the value of $\Delta=0.999$. In order to facilitate the discussion of the physical reasons for the observed features of the return time, we indicated three regimes for the curve with $\Delta=0$, as shown by the Roman numerals (I), (II) and (III). See text for a detailed discussion of the behavior of the return time in these regimes. Inset contains the same data plotted on semilog $(y)$ axes, and shows that the tail of the distributions are exponential functions.

calculating the probability with which this variable takes on positive (negative) values. The details of this calculation can be found in ref. [33]. Note that $f_{\mathrm{r}}$ is independent of $f$, and is determined by how many sites there are in a compartment and the hopping rate between sites. Therefore, in the strong confinement limit, the return time distribution can be expressed as

$$
P_{\mathrm{r}}(t)=\left(1-\frac{f}{f_{\mathrm{r}}}\right) P_{0}(t)+\frac{f}{f_{\mathrm{r}}} P_{1}(t ; f) .
$$

The $n^{\text {th }}$ moment of $P_{\mathrm{r}}(t)$ is given by

$$
\left\langle t^{n}\right\rangle_{\mathrm{r}}=\left(1-\frac{f}{f_{\mathrm{r}}}\right)\left\langle t^{n}\right\rangle_{0}+\frac{f}{f_{\mathrm{r}}}\left\langle t^{n}\right\rangle_{1},
$$

where $\left\langle t^{n}\right\rangle_{i}$ is the $n^{\text {th }}$ moment of the distribution $P_{i}(t)$. As $P_{1}(t)$ is the return time distribution for a random walk in a lattice of $N$ sites with waiting time distribution approximated by $f e^{-f t}$, we can use the results derived by Montroll and Weiss [21] to obtain

$$
\langle t\rangle_{1}=\frac{N}{f},
$$




$$
\left\langle t^{2}\right\rangle_{1}=2 N \frac{N \phi(N)+1}{f^{2}},
$$

where $\phi(N)$ is a sum whose value as $N \rightarrow \infty$ can be approximated by $2 N / 3$ (see ref. [21] equation II.29). Therefore, mean and variance of the return time distribution can be expressed as

$$
\begin{aligned}
& \mu_{\mathrm{r}}=\langle t\rangle_{\mathrm{r}}=\frac{f_{\mathrm{r}}\langle t\rangle_{0}+N}{f_{\mathrm{r}}}-\frac{f\langle t\rangle_{0}}{f_{\mathrm{r}}}, \\
& \sigma_{\mathrm{r}}^{2}=\left\langle t^{2}\right\rangle_{\mathrm{r}}-\langle t\rangle_{\mathrm{r}}^{2}=\frac{2 N(N \phi(N)+1)}{f_{\mathrm{r}} f}+\left(1-\frac{f}{f_{\mathrm{r}}}\right)\left\langle t^{2}\right\rangle_{0}-\mu_{r}^{2},
\end{aligned}
$$

Note that as the confinement strength increases further, i.e. $f \rightarrow 0$, the mean return time does not diverge, consistent with our other findings. In contrast, the variance of the return time keeps increasing without bound, due to the first term in the right hand side of equation (32), even in a finite lattice. Note that for the case of complete confinement, i.e. $f=0$, equation (28), and not (31) or (32), should be used to obtain the correct moment.

3.1.1. Taking the inverse Laplace transform of $\widetilde{Q}(\epsilon)$ In order to display the full firstpassage time distribution in time, we need to take the inverse Laplace transform of equation (3). After solving the linear system in equation (8) for $X$, we can calculate $\widetilde{P}\left(\epsilon, \vec{r} ; t=0, \vec{r}_{0}\right)$ and $\widetilde{P}(\epsilon, \vec{r} ; t=0, \vec{r})$ (see equation (6)), and finally the Laplace transform of the first-passage time distribution $\widetilde{Q}(\epsilon)$, as given by equation (3). There is no straightforward way to take the inverse Laplace transform of $\widetilde{Q}(\epsilon)$ by analytical methods. Therefore, we use a numerical method to invert the Laplace transform.

We pick the Gaver-Stehfest method $[34,35]$ because it only requires the evaluation of the Laplace transform at real values of the Laplace transform variable, and is reliable when the inverse transform is a bounded function such as the distribution of first-passage times. Employing the algorithm described by Abate and Whitt [36], we can approximate the inverse Laplace transform of $\widetilde{Q}(\epsilon)$ by

$$
\begin{aligned}
& Q(t)=\frac{\ln 2}{t} \sum_{k=1}^{2 M} w_{k} \widetilde{Q}\left(k \frac{\ln 2}{t}\right), \\
& w_{k}=(-1)^{M+k} \sum_{j=\lfloor(k+1) / 2\rfloor}^{\min (k, M)} j^{M+1} \frac{2 j !}{(j !)^{2}(M-j) !(2 j-k) !(k-j) !},
\end{aligned}
$$

where $M$ is a positive integer and $\lfloor(k+1) / 2\rfloor$ means the largest integer less than or equal to $(k+1) / 2$. The number $M$ is chosen based on the available numerical precision. According to an estimate given in reference [36], the calculation is reliable with $2.2 \mathrm{M}$ digits of precision.

In figure 3, the distribution of return times, obtained by averaging $Q(t)$ over all adjacent random walker-target configurations and denoted by $Q_{\mathrm{r}}(t)$, is plotted for $N=25$ and $H=4$, for different confinement strengths. To ensure the accuracy of results, the calculation was performed with 58 digits of precision and $M=20$. The axes 
in the main figure are both logarithmic to show the features of the curves over 6 decades in time (dimensionless). The inset shows the same data plotted on semilogarithmic axes $(y)$, and indicates that the tail of the distributions decay exponentially, with an exponent that is inversely proportional to confinement strength.

In order to discuss the features of the return time distribution, we will focus on the case of strong confinement (green curve in figure, with $\Delta=0.999$ ), and explain its behavior in physical terms, over three regimes indicated by the Roman numerals (I), (II) and (III) in figure 3. Behavior of the return time distribution for different confinement strengths is almost identical at short times because the effects of neither the compartment boundaries nor the finite size of the whole lattice are felt by the random walker when it finds the target before exploring most of the lattice (see regime I in figure 3). If there were no compartments and the lattice was infinite, we expect regime I to continue without giving way to an exponential decay, such that the mean return time is infinite, as it is well known for infinite, translationally invariant lattices [21] (also see equation (49) of the next section). At longer times, the features of the return time distribution significantly depend on the strength of confinement. As seen in regime II, in the presence of strong confinement, the return time distribution decays sharply after regime I, because the random walker quickly becomes well-mixed within the compartment that contains the target (due to the small escape rate that is proportional to $1-\Delta$ ). This sharp decay can be explained easily as the process of finding a target in a well-mixed system can be thought as Poisson process with a constant rate, giving rise to an exponentially decaying hitting time distribution. As the compartment boundaries are not perfectly reflecting, the random walker can escape without meeting the target. Even though the probability of occurrence of such an event is small, when it happens, it takes a long time before the random walker finds the target, as it searches in at least one empty compartment for a time that scales as $(1-\Delta)^{-1}$. For the case with $\Delta=0.999$, this part of the return time distribution is denoted by regime III. As the lattice is finite, regime III eventually ends with an exponential decay, reflecting the fact that if the random walker cannot find the target for a long time, it can be thought as a particle that is uniformly distributed over the lattice, and the arguments presented for regime II follow. Note that the time at which the final exponential decay begins is proportional to the confinement strength $\Delta$, as the time is takes for the system to become well-mixed is inversely proportional to the transfer rate between compartments, given by $1-\Delta$.

\subsection{A finite 2D lattice with reflecting boundaries}

In this section, we consider a 2D lattice of $M^{2}$ sites with reflecting boundaries and calculate the mean and variance of the first-passage time, again for a random walk with jumps between neighbouring sites. We present our results for the first arrival time of a mobile walker on an immobile target. Contrary to the previous section, here we treat time as a discrete variable. However, the results obtained for discrete time can be translated into their continuous time analogues as we will note at the end of 
this section. We would like to note that the probability propagator for a lattice with reflecting boundaries can in be principle obtained by using methods other than the one explained here, such as the method of images and discrete Fourier transform [32].

Let $\vec{r}$, and $\vec{r}_{0}$ be vectors containing the parameters that fully describe the state of a system at times $t$ and 0 , respectively. We denote the probability of finding the system in state $\vec{r}$ at time $t$, given it was in state $\vec{r}_{0}$ at $t=0$ by $P\left(\vec{r}, t \mid \vec{r}_{0}, 0\right)$. Here, we are interested in calculating the probability $F\left(\vec{r}, t \mid \vec{r}_{0}, 0\right)$ of finding the system in state $\vec{r}$ for the first time at $t$, given it was in state $\vec{r}_{0}$ at $t=0$. These two probabilities are related by

$$
P\left(\vec{r}, t \mid \vec{r}_{0}, 0\right)=\delta_{t, 0} \delta_{\vec{r}, \vec{r}_{0}}+\sum_{u=1}^{t} F\left(\vec{r}, u \mid \vec{r}_{0}, 0\right) P(\vec{r}, t \mid \vec{r}, u),
$$

which simply states that $P\left(\vec{r}, t \mid \vec{r}_{0}, 0\right)$ is the probability that the system first arrives at state $\vec{r}$ at time $u$, and is still found in that state at time $t$. It is more convenient to work with the generating functions for $F$ and $P$, given by

$$
\begin{aligned}
& P\left(\vec{r} \mid \vec{r}_{0}, z\right)=\sum_{t=0}^{\infty} z^{t} P\left(\vec{r}, t \mid \vec{r}_{0}, 0\right), \\
& F\left(\vec{r} \mid \vec{r}_{0}, z\right)=\sum_{t=1}^{\infty} z^{t} F\left(\vec{r}, t \mid \vec{r}_{0}, 0\right) .
\end{aligned}
$$

If we multiply both sides of equation (34) by $z^{t}$, sum over $t$ from 0 to $\infty$, and rearrange terms, we get [21]

$$
F\left(\vec{r} \mid \vec{r}_{0}, z\right)=\frac{P\left(\vec{r} \mid \vec{r}_{0}, z\right)-\delta_{\vec{r}, \vec{r}_{0}}}{P(\vec{r} \mid \vec{r}, z)}
$$

Note that equation (37) provides us with a way for calculating $F\left(\vec{r} \mid \vec{r}_{0}, z\right)$ in any system for which we know the probability distribution $P\left(\vec{r}, t \mid \vec{r}_{0}, 0\right)$. Here, the first system we consider consists of a random walker and a fixed target (a single lattice site) in a finite 2D lattice with reflecting boundary conditions. Therefore, $F\left(\vec{r}, t \mid \vec{r}_{0}, 0\right)$ is the first passage time distribution, for a random walker that starts at site $\vec{r}_{0}=\left(m_{0}, n_{0}\right)$ and arrives at $\vec{r}=(m, n)$ for the first time at time $t$. In the following, we show the $P\left(\vec{r}, t \mid \vec{r}_{0}, 0\right)$ in a confined domain can be constructed from its counterpart in a finite lattice with periodic boundary conditions.

Random walk in a lattice with reflective boundary conditions can be reduced to random walk in a periodic lattice of larger size. We start our discussion by considering the $1 \mathrm{D}$ case, which is simpler to illustrate. In a 1D lattice with periodic boundary conditions, i.e. a ring of $N$ lattice sites, the probability $P\left(m, t \mid m_{0}, 0\right)$ evolves according to

$$
P\left(m, t+1 \mid m_{0}, 0\right)=\frac{1}{2} P\left(m+1, t \mid m_{0}, 0\right)+\frac{1}{2} P\left(m-1, t \mid m_{0}, 0\right),
$$

which simply states that a particle moves to an adjacent lattice site with probability $1 / 2$ at each time step. Solving equation (38) for the generating function of $P\left(m, t \mid m_{0}, 0\right)$, see equation (35), by discrete Fourier transformation gives

$$
P\left(m \mid m_{0}, z\right)=\frac{1}{N} \sum_{k} \frac{e^{-i k\left(m-m_{0}\right)}}{1-z \cos k},
$$


where $k=2 \pi \ell / N, \ell=1,2, \ldots, N$. Using equations (39) and (37) we can calculate the first passage time distribution and its moments. See Montroll and Weiss [21] for a detailed discussion and results.

Here, we describe how these previously known results for periodic lattices can be used to construct the probability function for a lattice with reflecting boundaries. Note that the reflective boundary condition we consider here implies that when the random walker attempts to cross a boundary, it is reflected back, so that it moves at all time steps without the possibility of waiting at a boundary site. Suppose that we squeeze a ring, so that sites 2 to $N / 2$ are adjacent to sites $N / 2+2$ to $N$, as illustrated in figure 1(b). Next, we merge the adjacent pairs of sites, which amounts to summing up the probability of occupancy of these sites, so that we are left with a new lattice with only $M=(N+2) / 2$ sites. From now on, we denote probability of finding the random walker at the $m^{t h}$ site of the new lattice by $P_{c}\left(m, t \mid m_{0}, 0\right)$, where $1<m, m_{0}<M$ and the subscript $c$ indicates that the random walker is confined between two reflective boundaries. Putting all these together, the transformation of probabilities from the original periodic lattice to the final lattice with reflective boundary conditions becomes

$$
P_{c}\left(m, t \mid m_{0}, 0\right)=P\left(m, t \mid m_{0}, 0\right)+\alpha(m) P\left(N+2-m, t \mid m_{0}, 0\right),
$$

where $\alpha(m)=0$ for $m=1, M$ and 1 otherwise. Note that the boundary points are not affected by the transformation. This result can be readily extended to $2 \mathrm{D}$. In $2 \mathrm{D}$, the periodic lattice is a torus instead of a ring. It is convenient to think that each site on a torus belongs to two rings that extend along the two independent coordinates. As illustrated in figure 1(c), we need to apply the transformation we used in the 1D case two times for each point on the torus, such that a maximum of 4 sites may be merged. The transformation in $2 \mathrm{D}$ becomes

$$
\begin{aligned}
P_{c}\left(\vec{r}, t \mid \vec{r}_{0}, 0\right) & =P\left(\vec{r}, t \mid \vec{r}_{0}, 0\right)+\alpha_{1}(\vec{r}) P\left(N+2-m-m_{0}, N+2-n-n_{0}, t \mid m_{0}, n_{0}, 0\right) \\
& +\alpha_{2}(\vec{r}) P\left(m, N+2-n-n_{0}, t \mid m_{0}, n_{0}, 0\right) \\
& +\alpha_{3}(\vec{r}) P\left(N+2-m-m_{0}, n, t \mid m_{0}, n_{0}, 0\right),
\end{aligned}
$$

where $\alpha_{1}=1$ for $m \neq 1, M$ and $n \neq 1, M, \alpha_{2}=1$ for $m=1, M$ and $n \neq 1, M$, and finally $\alpha_{3}=1$ for $m \neq 1, M$ and $n=1, M$; all of them are equal to 0 otherwise. In other words, in the final lattice with reflective boundary conditions, if the site $(m, n)$ is at a corner, $\alpha_{1}=\alpha_{2}=\alpha_{3}=0$, if it is on one of the boundaries but not at a corner, $\alpha_{1}=0$, $\alpha_{2}$ or $\alpha_{3}$ is equal to 1 depending on the boundary, and if it is away from the boundaries $\alpha_{1}=\alpha_{2}=\alpha_{3}=1$. Note that $\alpha_{i}$ does not depend on the initial conditions.

The generating function for $P_{c}\left(\vec{r}, t \mid \vec{r}_{0}, 0\right)$ is simply the generalization of equation (39) to $2 \mathrm{D}$, and is given by

$$
P_{c}\left(\vec{r} \mid \vec{r}_{0}, z\right)=\sum_{\vec{k}} \frac{\xi\left(\vec{r}, \vec{r}_{0}, \vec{k}\right)}{1-z\left(\cos k_{1}+\cos k_{2}\right) / 2},
$$

where $\vec{k}=\left(k_{1}, k_{2}\right), k_{i}=2 \pi \ell / N, \ell=1,2, \ldots, N$, and

$\xi\left(\vec{r}, \vec{r}_{0}, \vec{k}\right)=\frac{1}{N^{2}}\left[e^{-i k_{1}\left(m-m_{0}\right)-i k_{2}\left(n-n_{0}\right)}+\alpha_{1} e^{-i k_{1}\left(2-\left(m+m_{0}\right)\right)-i k_{2}\left(2-\left(n+n_{0}\right)\right)}\right.$ 


$$
\left.+\alpha_{2} e^{-i k_{1}\left(m-m_{0}\right)-i k_{2}\left(2-\left(n+n_{0}\right)\right)}+\alpha_{3} e^{-i k_{1}\left(2-\left(m+m_{0}\right)\right)-i k_{2}\left(n-n_{0}\right)}\right] .
$$

Substituting the above expression for $P_{c}\left(\vec{r} \mid \vec{r}_{0}, z\right)$ in equation (37), we obtain the generating function for the first-passage time distribution $F_{c}\left(\vec{r} \mid \vec{r}_{0}, z\right)$. The generating function $F_{c}\left(\vec{r} \mid \vec{r}_{0}, z\right)$ is related to $F_{c}\left(\vec{r}, t \mid \vec{r}_{0}, 0\right)$ as follows

$$
F_{c}\left(\vec{r}, t \mid \vec{r}_{0}, 0\right)=\left.\left(\frac{1}{t !} \frac{d^{t}}{d z^{t}} F_{c}\left(\vec{r} \mid \vec{r}_{0}, z\right)\right)\right|_{z \rightarrow 0} .
$$

The mean and variance of this distribution can be conveniently calculated, using the definition in equation (36), and are found to be

$$
\begin{aligned}
& \mu_{\overrightarrow{\mathrm{r}}_{0}, \overrightarrow{\mathrm{r}}}=\left.\left(\frac{d}{d z} F_{c}\left(\vec{r} \mid \vec{r}_{0}, z\right)\right)\right|_{z \rightarrow 1}, \\
& \sigma_{\overrightarrow{\mathrm{r}}_{0}, \overrightarrow{\mathrm{r}}}^{2}=\left.\left(\frac{d^{2}}{d z^{2}} F_{c}\left(\vec{r} \mid \vec{r}_{0}, z\right)\right)\right|_{z \rightarrow 1}+\mu_{\overrightarrow{\mathrm{r}}_{0}, \overrightarrow{\mathrm{r}}}-\mu_{\overrightarrow{\mathrm{r}}_{0}, \overrightarrow{\mathrm{r}}}^{2},
\end{aligned}
$$

which are explicitly given by

$$
\begin{aligned}
\mu_{\overrightarrow{\mathrm{r}}_{0}, \overrightarrow{\mathrm{r}}} & =\frac{\delta_{\vec{r}_{0}, \vec{r}}}{\xi_{0}(\vec{r})}+\frac{1}{\xi_{0}(\vec{r})}\left(\beta_{\vec{r}, \vec{r}}^{(0)}-\beta_{\vec{r}, \vec{r}_{0}}^{(0)}\right), \\
\sigma_{\overrightarrow{\mathrm{r}}_{0}, \overrightarrow{\mathrm{r}}}^{2} & =\left(\frac{2 \beta_{\vec{r}, \vec{r}_{0}}^{(0)}}{\xi_{0}(\vec{r})^{2}}+\mu_{\overrightarrow{\mathrm{r}}_{0}, \overrightarrow{\mathrm{r}}}-\mu_{\overrightarrow{\mathrm{r}}_{0}, \overrightarrow{\mathrm{r}}}^{2}\right) \delta_{\vec{r}_{0}, \vec{r}}+\frac{1}{\xi_{0}(\vec{r})^{2}}\left(\left[\beta_{\vec{r}, \vec{r}}^{(0)}\right]^{2}-\left[\beta_{\vec{r}, \vec{r}_{0}}^{(0)}\right]^{2}\right) \\
& +\frac{1}{\xi_{0}(\vec{r})}\left(\beta_{\vec{r}, \vec{r}}^{(0)}-\beta_{\vec{r}, \vec{r}_{0}}^{(0)}+2\left[\beta_{\vec{r}, \vec{r}}^{(1)}-\beta_{\vec{r}, \vec{r}_{0}}^{(1)}\right]\right),
\end{aligned}
$$

where

$$
\begin{aligned}
& \beta_{\vec{r}, \vec{r}_{0}}(z)=\sum_{\vec{k} \neq(2 \pi, 2 \pi)} \frac{\xi\left(\vec{r}, \vec{r}_{0}, \vec{k}\right)}{1-z\left(\cos k_{1}+\cos k_{2}\right) / 2}, \\
& \beta_{\vec{r}, \vec{r}_{0}}^{(n)}=\lim _{z \rightarrow 1} \frac{d^{n}}{d z^{n}} \beta_{\vec{r}, \vec{r}_{0}}(z), \\
& \xi_{0}(\vec{r})=N^{-2}\left(1+\alpha_{1}(\vec{r})+\alpha_{2}(\vec{r})+\alpha_{3}(\vec{r})\right) .
\end{aligned}
$$

In order to test the validity of equations (45) and (46), we compared them against Monte Carlo simulations for five randomly chosen initial and final position pairs $\left(\vec{r}_{0}, \vec{r}\right)$ in a $5 \times 5$ lattice. In the simulations, the random walker starts at site $\vec{r}_{0}$, and hops between adjacent lattice sites, at each time step. If the walker is at a boundary, the probability of moving perpendicular to the boundary is twice that of moving along the boundary, such that if it attempts to cross the boundary, it is reflected. The comparison between simulation results and predictions given in equations (45) and (46) are presented in table 1, which shows perfect agreement. Note that a similar result for the first-passage time was given earlier by Condamin et al. [26] for a rectangular lattice; however, the boundary conditions employed in that study allows the random walker to wait at the boundary during a time step, giving rise to first-passage time statistics that is different from what we find here, for finite lattices.

In particular, the mean and variance of the return time, that is the time it takes for the particle to return to its starting point averaged over all initial positions, are given 


\begin{tabular}{|c|c|c|c|c|}
\cline { 2 - 5 } \multicolumn{1}{c|}{} & \multicolumn{2}{c|}{$\mu_{\vec{r}_{0}, \vec{r}}$} & \multicolumn{2}{c|}{$\sigma_{\vec{r}_{0}, \vec{r}}^{2}$} \\
\hline$\vec{r}_{0}, \vec{r}$ & eqn. (45) & sim. & eqn. (46) & sim. \\
\hline$(5,2),(4,5)$ & 58.1 & $58.2 \pm 0.4$ & 3110.4 & $3134.5 \pm 79.7$ \\
\hline$(2,1),(2,3)$ & 19.8 & $19.7 \pm 0.2$ & 511.2 & $503.8 \pm 14.3$ \\
\hline$(5,2),(3,1)$ & 42.1 & $42.3 \pm 0.6$ & 2149.4 & $2168.8 \pm 68.3$ \\
\hline$(4,1),(2,4)$ & 64.2 & $64.3 \pm 0.7$ & 3149.2 & $3165.2 \pm 91.5$ \\
\hline$(5,3),(5,2)$ & 37.3 & $37.1 \pm 0.4$ & 2681.1 & $2651.3 \pm 77.7$ \\
\hline
\end{tabular}

Table 1. To confirm the validity of the mean and variance of the first-passage time given in equations (45) and (46), we compared them against Monte Carlo simulations of lattice walks, for five randomly chosen initial and final points in a $5 \times 5$ lattice. See text for the details of the simulation algorithm, especially concerning how particles at the boundary move. These results show that theory and simulations agree within statistical limits (the values given after \pm signs correspond to the standard deviation of simulation results).

by

$$
\begin{aligned}
\mu_{\mathrm{r}} & =\frac{1}{M^{2}} \sum_{\vec{r}_{0}} \frac{1}{\xi_{0}\left(\vec{r}_{0}\right)}=\frac{(M-1)^{2}(M+2)^{2}}{M^{2}}=M^{2}+2 M-3-\frac{4}{M}+\frac{4}{M^{2}} \\
\sigma_{\mathrm{r}}^{2} & =\frac{1}{M^{2}} \sum_{\vec{r}_{0}}\left(\frac{2 \beta_{\vec{r}_{0}, \vec{r}_{0}}^{(0)}}{\xi_{0}\left(\vec{r}_{0}\right)^{2}}+\mu_{\overrightarrow{\mathrm{r}}_{0}, \overrightarrow{\mathrm{r}}_{0}}-\mu_{\overrightarrow{\mathrm{r}}_{0}, \overrightarrow{\mathrm{r}}_{0}}^{2}\right) \\
& =\frac{2}{M^{2}} \sum_{\vec{r}_{0}} \frac{\beta_{\vec{r}_{0}, \vec{r}_{0}}^{(0)}}{\xi_{0}\left(\vec{r}_{0}\right)^{2}}-\frac{(M-1)^{4}(M+6)^{2}}{M^{2}}+\mu_{\mathrm{r}} .
\end{aligned}
$$

To check the validity of the mean and variance of the reencounter (return) time, we again compared them against results obtained by Monte Carlo simulations. In figure 4, we display the comparison between simulation results and equations (47) and (48) for small lattices of up to 100 sites, which confirms the validity of theoretical predictions.

In a d-dimensional lattice with $M^{d}$ sites and periodic boundary conditions, the mean and variance of the return time are given by [21]

$$
\begin{aligned}
& \mu_{\mathrm{r}, \mathrm{per}}=M^{d}, \\
& \sigma_{\mathrm{r}, \mathrm{per}}^{2}=2 M^{d} \sum_{\vec{k}}^{\prime}\left(1-\frac{1}{d} \sum_{i=1}^{d} \cos \left(2 \pi k_{i} / M\right)\right)^{-1}+M^{d}\left(1-M^{d}\right),
\end{aligned}
$$

where $\vec{k}=\left(k_{1}, k_{2}, k_{3}, \ldots, k_{d}\right), k_{i}=1,2,3, \ldots, M$, and the prime indicates that the value of $\vec{k}$ for which the denominator of the summand becomes 0 is excluded.

Finally, we would like to note that if we treat the motion of the particles as a continuous time random walk with exponential waiting time $\tau$, the mean and variance for the continuous return time will be related to the results above by [21]

$$
\begin{aligned}
& \mu_{\mathrm{r}}^{\prime}=\tau \mu_{\mathrm{r}} \\
& \sigma_{\mathrm{r}}^{\prime 2}=\tau^{2}\left(\sigma_{\mathrm{r}}^{2}+\mu_{\mathrm{r}}\right) .
\end{aligned}
$$




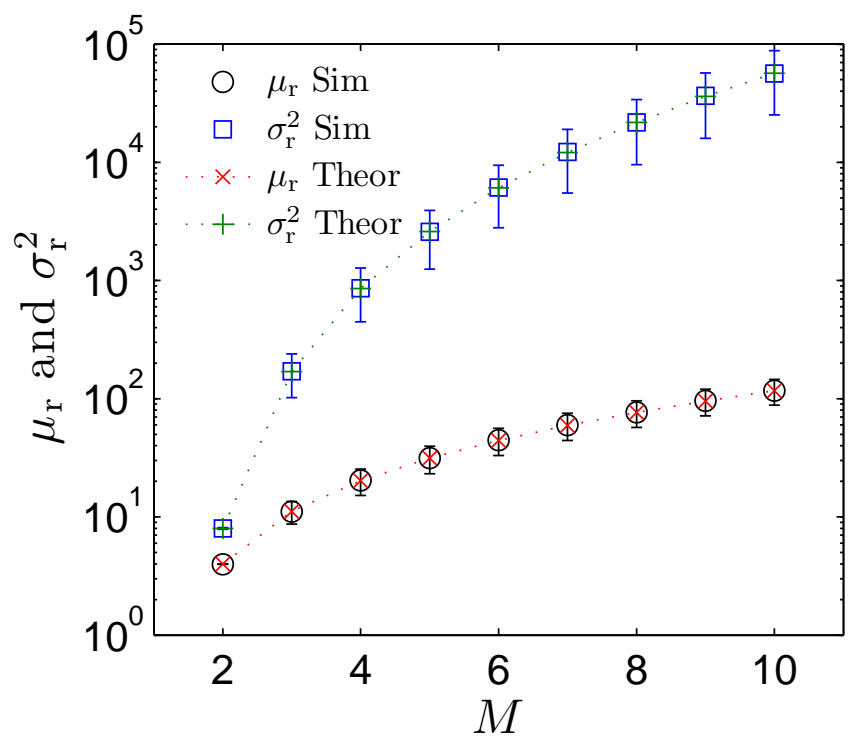

Figure 4. Mean and variance of the return time, as a function of lattice size, obtained by Monte Carlo simulations and via equations (47) and (48). Results show remarkable agreement between the theory and simulations. For the simulation results, error bars indicate the standard deviation around the mean. Given the number of simulation runs per initial condition was sufficiently large $\left(10^{4}\right)$, the variation around the mean is almost entirely due to different initial conditions. Note that the error bars are asymmetric as the $y$ axis is logarithmic.

See Montroll and Weiss [21] for more general relations valid for arbitrary waiting time distributions.

\section{Discussion and conclusions}

In summary, we presented solutions for two first-passage time problems for lattice walks in the presence of temporarily or completely confining domains. For a single random walker jumping between adjacent lattice sites in a finite 1D lattice partitioned into temporarily confining domains by periodically placed permeable barriers, we gave exact formulas for the mean and variance of the first-passage time from an arbitrary site to a target site, and the return (reencounter) time distribution. One of our interesting findings is that the mean return time weakly depends on the confinement strength, which is inversely proportional to the rate of crossing a barrier; whereas its variance increases without bound (see figure 2). This may have interesting implications for reversible reactions that take place in a partitioned medium. For reactions such as reversible dimerization, the time it takes before the reactants reencounter following a dissociation controls the time between reaction events, hence the reaction rate. In our case, the mean return time is almost independent from the confinement strength, implying that the average forward rate of reversible reactions between a random walker and a target site does not depend on confinement strength. However, the variance of 
the reaction rate would strongly depend on confinement strength. Therefore, ensemble averaged measurements of reaction rates may not reveal any features of the structure of the medium; however, single particle level observations that are able to measure the variance can. Furthermore, by using a numerical method to invert Laplace transforms, we presented the full first-passage time distribution for this case, and compared it with the analogous distribution in a translationally invariant lattice that does not contain any permeable barriers (see figure 3 ).

In a finite $2 \mathrm{D}$ lattice with reflective boundaries, i.e. complete confinement, we gave exact results for the mean and variance of the first-passage time from an arbitrary site to a target, as well as the return time. We obtained these results by finding a relation between the lattice Green's functions for a translationally invariant periodic lattice and that for a finite lattice with reflective boundaries (see equations (40) and (41)). It is worth noting that when reactants are mobile, obtaining exact solutions under general conditions is a formidable task. Nevertheless, Moreau et al. [37] gave generally valid results for the upper bound of the time to reaction when the reactants are mobile, in a system consisting of many interacting random walkers. In a translationally invariant periodic lattice, a system of two mobile walkers can be thought as a single walker that jumps twice as frequently in the presence of fixed targets, as discussed in Abad et al. [38], and Abad [39]. For a detailed discussion on this, and its generalization to random walks in disordered networks, see García Cantú and Abad [40]. In higher dimensions, a precise relationship between the return time and the properties of spatial heterogeneities such as confining domains, or the presence of other interacting walkers [37] has still not been found, and is worth investigating.

Effects of confinement on other aspects of molecular interactions in a lattice has been the subject of several other recent studies as well. In a series of works, Kozak and collaborators studied bimolecular reactions on a lattice, with periodic or confining boundary conditions, by considering the motion of two independent random walkers $[13,15,17]$. The main quantity of interest in their studies was the average number of steps that the random walkers take before an encounter. One of their findings is that for small, confined, systems, i.e. when the particles can rapidly explore most of the space available to them, counter intuitive regimes may be observed in which reaction efficiency may decrease with increasing particle mobility [18]. Furthermore, the presence of a large number of inert particles is a significant and physically meaningful factor that can influence reaction rates. For instance, Schmit et al. [41] considered a lattice walk model and predicted that the rate of bimolecular diffusion limited reactions in confined, crowded environments can behave non-monotonically. A series of remarkable results about the effect of confining geometries on the first-passage times were obtained by Condamin et al. [25, 22, 26], who also discussed the effects of having multiple targets, and considering random walks in continuum.

In our future studies, we would like to develop a basic theory of reversible molecular associations in a heterogeneous medium, partitioned into transiently confining domains, based on a stochastic study of the spatio-temporal distribution of molecular encounters 
at the single molecule level. Such a theory is much needed in studying mesoscopic phenomena in soft matter, especially when the number density of interacting molecules is low. We believe that the conceptual study of chemical reactions and the formation of dynamic, functional molecular complexes could greatly benefit from the development of a theoretical framework along these lines.

\section{Acknowledgments}

I would like to thank Akihisa Otaka for his help with writing computer programs employing high precision arithmetics, and the two anonymous reviewers for their critical comments. Thanks to Ikumi for her support and endless patience. This research was supported in part by the World Premier International Research Center (WPI) Initiative of the Ministry of Education, Culture, Sports, Science and Technology (MEXT) of Japan.

\section{References}

[1] Redner S 2001 A Guide to First-Passage Processes (Cambridge, UK: Cambridge University Press)

[2] von Smoluchowski M 1917 Z. Physik. Chem. 92129

[3] Noyes R M 1954 J. Chem. Phys. 221349

[4] Kenkre V M and Reineker P 1982 Exciton Dynamics in Molecular Crystals and Aggregates (Springer Tracts in Modern Physics no 94) (Berlin: Springer-Verlag)

[5] Hänggi P, Talkner P and Borkovec M 1990 Rev. Mod. Phys. 62 251-341

[6] Singpurwalla N D 1995 Stat. Sci. 10 86-103

[7] Shlesinger M F 2006 Nature 443 281-282

[8] Collins F C and Kimball G E 1949 J. Colloid Sci. 4 425-437

[9] Naqvi K R, Mork K J and Waldenstrom S 1980 J. Phys. Chem. 84 1315-1319

[10] Agmon N and Szabo A 1990 J. Chem. Phys. 925270

[11] Van Beijeren H, Dong W and Bocquet L 2001 J. Chem. Phys. 1146265

[12] Torney D C and McConnell H M 1983 P. Roy. Soc. Lond. A Mat. 387 147-170

[13] Nicolis C, Kozak J J and Nicolis G 2001 J. Chem. Phys. 115663

[14] Musho M K and Kozak J J 1984 J. Chem. Phys. 80159

[15] Kozak J J, Nicolis C and Nicolis G 2001 J. Chem. Phys. 1138168

[16] Garza-Lopez R A, Kaufman A, Patel R, Chang J, Brzezinski J and Kozak J J 2008 Chem. Phys. Lett. 459 137-140

[17] Bentz J L, Kozak J J, Abad E and Nicolis G 2003 Physica A 326 55-68

[18] Abad E and Kozak J J 2006 Physica A 370 501-524

[19] Saxton M J 2008 Biophys. J. 94 760-771

[20] Saxton M J 2002 J. Chem. Phys. 116 203-208

[21] Montroll E W and Weiss G H 1965 J. Math. Phys. 6167

[22] Condamin S, Bénichou O, Tejedor V, Voituriez R and Klafter J 2007 Nature 450 77-80

[23] Bénichou O, Chevalier C, Klafter J, Meyer B and Voituriez R 2010 Nat. Chem. 2 472-477

[24] Meyer B, Chevalier C, Voituriez R and Bénichou O 2011 Phys. Rev. E 83051116

[25] Condamin S, Benichou O and Moreau M 2005 Phys. Rev. Lett. 95260601

[26] Condamin S, Benichou O and Moreau M 2007 Phys. Rev. E 75021111

[27] Aldous D and Fill J 1999 Reversible Markov Chains and Random Walks on Graphs (http://www.stat.berkeley.edu/users/aldous/RWG/book.html)

[28] Mejía-Monasterio C, Oshanin G and Schehr G 2011 J. Stat. Mech. P06022 
[29] Tejedor V, Schad M, Bénichou O, Voituriez R and Metzler R 2011 J. Phys. A 44395005

[30] Doetsch G 1971 Guide to the Applications of the Laplace and z-Transforms (London: Van Nostrand Reinhold Co. Ltd.)

[31] Kenkre V M, Giuggioli L and Kalay Z 2008 Phys. Rev. E 77051907

[32] Hughes B D 1995 Random Walks and Random Environments (Oxford: Clarendon Press)

[33] Kalay Z, Fujiwara T K and Kusumi A 2012 PLoS ONE 7 e32948

[34] Gaver D P 1966 Oper. Res. 14 444-459

[35] Stehfest H 1970 Commun. ACM 13 47-49

[36] Abate J and Whitt W 2006 INFORMS J. Comput. 18 408-421

[37] Moreau M, Oshanin G, Bénichou O and Coppey M 2004 Phys. Rev. E 69046101

[38] Abad E, Nicolis G, Bentz J L and Kozak J J 2003 Physica A 326 69-87

[39] Abad E 2005 Phys. Rev. E $\mathbf{7 2} 021107$

[40] García Cantú A and Abad E 2008 Phys. Rev. E 77031121

[41] Schmit J D, Kamber E and Kondev J 2009 Phys. Rev. Lett. 102218302 